\title{
Mind-Matter Entanglement Correlations: Blind Analysis of a New Correlation Matrix Experiment
}

\author{
Hartmut Grote \\ Max Planck Institute for Gravitational Physics, Hannover, Germany \\ Submitted August 6, 2020; Accepted January 4, 2021; Published June 15, 2021 \\ https://10.31275/20211931 \\ Creative Commons License CC-BY-NC
}

Abstract-The work reported here is a rigorous conceptual replication of the so-called "Correlation-Matrix" experiment by an independent author. The experiment has been built from scratch with new hardware and software, testing 200 participants who have spent about half an hour each trying to 'influence' a physical random process visualized for feedback. The analysis software has been conceptualized following a strict blind analysis protocol. Blind analysis is a more rigid form of pre-registered analysis, in which the complete analysis software is written and tested before the data are actually analyzed for the effect under study. The unblinding of the analysis, also called the 'opening of the box', of the experiment described here was performed live at the Parapsychological Association convention in 2019 in Paris. The main result was found not to be statistically significant and fell well within the expected random distribution of possible results. A second experiment, also following a blind analysis protocol, included questionnaires that were correlated with the participants' performance to 'influence' the physical random process (the main psi task). This yielded a probability of $p=.06$ to have occurred by chance, under a null hypothesis. A post hoc analysis of the hit rate for the psi task across all participants, which is mathematically independent from the correlation analysis, yielded a probability of $p=.06$ as well, to have occurred by chance. Three unexpected anecdotal incidents that occurred during the execution of the experiment and the testing and actual analysis of the data may add to the canon of oddities and trickster-like effects sometimes reported in parapsychology research.

Keywords: mind-matter, entanglement, parapsychology, Correlation Matrix 


\section{INTRODUCTION}

Mind-matter interaction or micro-psychokinesis (PK) experiments have a long tradition in parapsychology and, due to inherent difficulties in these experiments, often have become more complex in their nature over the years. One of these more complex developments was to use correlations between psychological variables of a human agent with actual physical variables of a system. For example, instead of looking for an aggregate deviation from randomness, one would correlate the output of a random number generator (RNG) with psychological traits such as belief in parapsychological phenomena, or others. An additional level of complexity was introduced by Walter von Lucadou by using many such correlations between different psychological and different physical variables within one experiment. Arranging all the resulting correlation factors in a matrix, he called this type of experiment the "Correlation Matrix Method"(CMM) (von Lucadou 1986, 1991, 2006). To evaluate statistical significance of a potential psi effect, all correlation factors in the matrix have to be evaluated together, as an ensemble.

The idea of the CMM method was created in conjunction with the hypothesis that psi may act in the form of correlations, rather than in the form of a causal signal transmission. In an analogy to physics, such hypothetical entanglement-like correlations could not be used to reliably transmit information, which would make it un predictable where in a given PK-type experiment significant correlations would show up. Thus, the combined result of many correlations is evaluated in a CMM-type experiment, without predicting or expecting any particular correlation to show significance. In essence, this is a multiple-analysis technique. The idea of a CMM-type experiment has also been related to the theoretical backgrounds of Generalized Quantum Theory (Filk \& Römer, 2011) and the model of pragmatic information (von Lucadou, 1995).

Von Lucadou performed three such CMM-type experiments and reported statistical significance for each of these (von Lucadou 1986, 1991, 2006), which has led lately led to a new replication involving von Lucadou and other researchers (Walach et al., 2020). There has been some debate about a statistically correct method to evaluate the significance of the ensemble of correlation factors in the matrix. ${ }^{1}$ At 
present, empirical methods (based on permutations and/or simulations of data) to estimate the statistical background distribution, as proposed and applied by Grote $(2015,2017)$, seem to be the only viable method. The reason for this is that in virtually all correlation matrix experiments performed to date, there are strong correlations between the psychological variables obtained, which renders all analytical statistical methods, which usually rely on statistical independence, invalid. The work by Walach et al. (2020) has followed this empirical approach to estimate the statistical significance and obtained less significant results than were reported by von Lucadou (1986, 1991, 2006).

In his two earlier experiments (von Lucadou, 1986, 1991), von Lucadou used questionnaires to obtain psychological variables before the physical variables, derived from RNG output, were obtained. In his later experiment (von Lucadou, 2006), as well as in the replication (Walach et al., 2020), this design was changed to using the choice of button pushes by the participants as psychological variables. These variables from button pushes were obtained during individual runs (see details below) and correlated to physical variables from RNG output of earlier runs, the same run, and later runs. The correlation of psychological variables to physical variables (which are presented as feedback to the participant and thus may influence the psychological variables) from earlier runs and the same run has led to some controversy about possible causal correlations. While there seems to be no hint of causal correlations in the data of the experiments described by von Lucadou (2006) and Walach et al. (2020), it seems more prudent to use only psychological variables that have been obtained before the physical variables, if truly non-causal correlations are to be elucidated and investigated.

In the study reported here, the choice was made for a 'buttonpush' scheme, as in the studies by von Lucadou (2006) and Walach et al. (2020), but to only use psychological variables obtained before physical ones for the main analysis. We call this 'Experiment 1 ' of this study, as submitted to the Bial Foundation. In addition to this experiment, each participant was asked to fill out a questionnaire before the apparatus-phase of the experiment. The questionnaires were planned to be correlated with the main RNG output across all participants, which we call 'Experiment 2'. 
While Experiment 1 described here is a close conceptual replication of a CMM experiment facilitating the basic experimental design as used by von Lucadou and others, some notable differences will be pointed out. Experiment 2 is a conceptual replication of the work reported in Jolij and Bierman (2019).

\section{METHODS}

\section{Experimental Apparatus}

The experimental apparatus has been designed and built by the author of this study. The choice was made to build a new, dedicated hardware device rather than using a standard computer, to give the experiment some feeling of uniqueness. The device consisted of a case made of wood and metal, with a small (7-inch) high-resolution video screen and four dedicated push buttons, as shown in Figure 1.

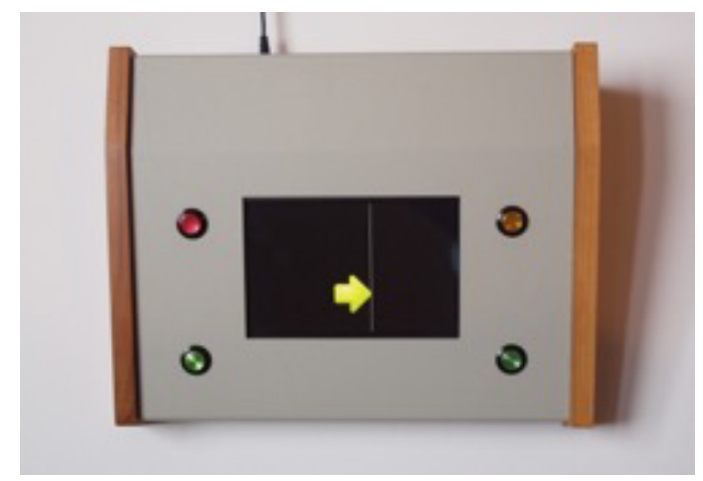

Figure 1. The experimental apparatus. The video screen shows an arrow the direction of motion of which was to be 'influenced' by the participant, while being driven by true random events.

\section{Random Event Generator}

The apparatus featured a new hardware random number generator developed by the author. The source of randomness was based on the thermal noise of two electrical resistors in a differential and shielded configuration that minimized coupling of environmental fluctuations to the noise currents of the resistors. Binary integer numbers of 16-bit lengths (called random Words) were obtained by 16 subsequent analogto-digital conversions of the noise current with 10-bit resolution. Each 
of these 16 conversions produced one bit by using only bit 7 of each sample, to be used as one bit of the 16-bit-long Word. To validate the quality of the random Words, they were scrutinized with Marsaglia's 'die-hard' suite of tests (Marsaglia, 1995). No suspicious deviation from randomness was found.

To generate random events for the purpose of the experiment, a new random Word would be drawn when required and compared with the previously drawn random Word. If the new Word was larger than the former, a logical ' 1 ' was defined as outcome. If the new Word was smaller than the former, a 'o' was defined. The procedure would be repeated in the unlikely event that the Words would be equal. This procedure is similar to a 1-step Markov chain and is the procedure that was used for the experiments described in von Lucadou (2006) and Walach et al. (2020). (Those experiments used single samples of noise of a Zener diode as inputs to the Markov chain.) We call the resulting bits 'Markov bits' or 'random events'.

\section{Two Types of Random Events}

For about half of the participants of the experiment, the above procedure was used to generate Markov bits in real time, at the time they were needed to progress the experiment. We call these bits RT-bits (Real Time). For the other half, the above procedure was modified in two ways: First, the random Words from the RNG were modified with an XOR operation with random (16-bit-long) Words from a pseudoRNG algorithm (Mersenne Twister, Matsumoto \& Nishimura, 1998), which was seeded with independently drawn random Words at the start of the experiment. Second, the full sequence of Markov bits was generated (using the XOR modified random Words) at the start of the experiment and then stored in the memory of the control computer. Data were read from this sequence when they were needed to progress the experiment at later times, typically over a period of 15-30 minutes after their generation. We call bits generated in this way XPR-bits (XOR'd and Pre-Recorded).

The split in these two types of random events was introduced to possibly test any result against these seemingly different sources of randomness. The XOR operation with a pseudo-RNG stream would 
test whether effects are robust against different complexity levels of the RNG, while the pre-recording aspect would test if correlations work 'backwards in time', also referred to as retrocausation. Work by Schmidt and others has mostly led to the hypothesis that neither of these modifications would affect results (Schmidt, 1976, 1987).

The analysis for Experiments 1 and 2 as defined in the preregistered blind analysis protocols did not make use of the distinction between the two RNG types, but the split was used in a secondary analysis, which was also performed in a blind, but not publicly preregistered, way.

\section{Experimental Sessions}

The sequence and tasks of the experimental sessions were very similar to the experiments as reported by von Lucadou (2006) and Walach et al. (2020), but the apparatus was novel, as reported above.

For each experimental session, a participant was first instructed about the general goals of the study, with the opportunity to ask questions. The study had been approved by the ethics committee of the Max Planck Society in Germany, and each participant signed a consent form allowing the anonymous use of their data.

In a next step, each participant was asked to fill out a 2-page questionnaire, comprising 50 questions to be answered on a 5 -point Likert scale. After this was completed, the participant was guided to the experimental apparatus located in a separate room on a desk in the given localities.

The participants were then asked to press a button to start the experiment. Upon this button press, the control computer would decide randomly (using the comparison of two random words) whether to use RT-bits or XPR-bits for this participant. The participants did not know of the two different bit-generation modalities, and the experimenter (the author) did not know which bit type was determined for each participant until removal of the blind condition.

A test run was then performed in the presence of the author (who was the sole experimenter for all participants), with the author explaining the conducting of the experiment on the hardware device. After this procedure, each participant was left alone in the room to 
perform the main experiment in their own time.

The participant had the task of trying to 'influence' the displayed arrow (see Figure 1 ) to move more in the direction that it was pointing to, either to the right-hand or to left-hand side, or to keep it in the middle of the display, when it was pointing down. A typical experimental session consisted of 9 'runs' for each participant. For each run the participant was asked to use the left and right hand to push a button with either hand, respectively (there was a button for the left hand and a button for the right hand). The possibility of pushing both buttons at the same time was left open and not deliberately mentioned. Each participant then had the task of pressing the buttons at a time of their choice with each button push triggering the generation of an RT-bit at that time, or the reading of an XPR-bit from memory. The obtained bit determined the direction of motion of an arrow in the display (right or left) by one step of fixed size. Each button push was accompanied by a 'cheering' sound when the arrow moved in the intended (intention according to the instruction given) direction (a hit), and by a less pleasant sound when it moved in the opposite direction (a miss). When a new record position of the arrow to the right or left was achieved within a run (with a minimum of 3 steps to either side), a longer 'cheering' sound from a cheering group of people was played back, intended as a particular reward. The auditory feedback was novel in this experiment, with the intent to strengthen the feedback experience.

The condition of the arrow to point right, down, or left was given in each run, thus setting the nominal intention of the participant deliberately for each run. Each of the three conditions (right, middle, left) occurred 3 times, for a total of 9 runs. A run would be finished after 80 button presses and a new run would be started by the participant at will, upon the pressing of a third (yellow) button.

After each run, short feedback was given in the form of a text (as in von Lucadou, 2006) and in the form of the achieved hit scores (for the right and left conditions combined). After the 9 runs a summary feedback was given in form of a text as well as the total hit score and the information that the experiment had now finished. 


\section{Data Recording}

For each button press ( 80 per run), data were stored on the nonvolatile memory of the control computer, namely:

- which button was pressed (left or right or both)

- the time of the button presses, with a resolution of less than 1 millisecond

— the raw, 16-bit-long Word from the 16 AD samples (for RT-bits) or the 16-bit-long Word from memory (for XPR-bits)

In addition to this, three 16-bit-long Words, and correspondingly three derived bits, were stored as control data that were sampled approximately $1 \mathrm{~ms}$ before ( $1 \mathrm{x}$ ) and $1 \mathrm{~ms}$ and $2 \mathrm{~ms}$ after (2x) the experimental sample as described above. These data were not planned to be used in any analysis, but to be used for consistency checks, as reported below.

\section{Pre-Data-Taking Registration}

The code running on the computer of the experimental apparatus, the feedback audio files, test random data, as well as the consent form and the questionnaire, have been pre-registered, prior to data-taking, on the open science framework platform and are publicly accessible at https://osf.io/cgf4k.

\section{Participants}

Participants were recruited from different pools. A total of 200 participants, the pre-planned number, completed the experiment in the period from December 2017 to May 2019. Of these, 75 were recruited from the general population in the Santa Fe area in New Mexico, USA, and performed the experiment at Mountain Cloud Zen Center in Sante Fe. 48 participants were drawn from a student population of various fields of study in Freiburg im Breisgau in Germany. They performed the experiment at the IGPP institute in Freiburg. A further 56 participants were recruited and tested in Berlin and Hannover in Germany, and the remaining 21 participants were recruited and tested in Oxford and Cardiff in the UK.

The average participant age was 45.6 years with a standard deviation 


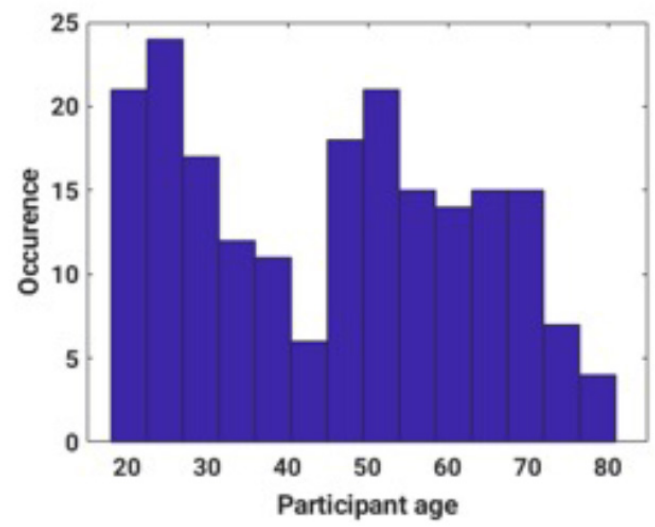

Figure 2. Age distribution across participants.

of 17.5 years. 78 participants reported as male and 122 as female. Figure 2 shows the age distribution of all participants.

\section{Blind Analysis Protocol}

Blind analysis is a strict form of a pre-defined analysis and is increasingly used in fields such as medicine and physics (Klein \& Roodman, 2005) to prevent bias in the analysis procedure of a dataset. While the social sciences and psychology use blind analysis less, these fields may often benefit from it (MacCoun \& Perlmutter, 2015). Potential bias in the data-analysis of parapsychological experiments has been discussed by Bierman et al. (2016) and Wagenmakers et al. (2015), among others.

In blind analysis, not only is the analysis plan pre-registered, but also the complete analysis algorithm, typically a computer program, which is written, fully debugged, and tested before the experimental data are analyzed for the effect under study. This ensures that detailed questions of the analysis that can affect the result (but that may show up only during the performance of the actual analysis) have to be decided beforehand, and any bugs associated with the analysis can be dealt with without introducing biases. In particular, during the 'blind' phase of the analysis development and testing, either artificial data are used, or the real experimental data is used, but in a disguised, i.e., blinded, form. The latter can be achieved conveniently in correlationtype experiments (such as this one) by permuting the association of 
psychological to physical variables between different participants. This was chosen for the development of the blind analysis codes for this experiment.

The analysis for this experiment had been specified and tested during the data acquisition phase. In particular the data had not been looked at or analyzed in any way before the unblinding, in agreement with the blind analysis protocol. The analysis of the experiment had been implemented in software (Matlab) as outlined below. The analysis code, as well as all the experimental data, have been pre-registered and are accessible at https://osf.io/xwhn6 for Experiment 1 and at https:// osf.io/cj8kd for Experiment 2.

For Experiment 1, the data-analysis procedure closely follows the way the data was analyzed for the latest experiments described in von Lucadou (2006), except for the evaluation of statistical significance of the matrix of correlation factors (which was performed as outlined below). The choice of variables resembles the choice of the experiment reported in Walach et al. (2020), with the exception that one of the psychological and physical variables each was replaced with new ones, as indicated below.

From the data recorded during the experiment (see above), the following five psychological and five physical variables were computed for each run:

Psychological variables:

- Number of left button presses

- Number of both button presses (simultaneous left and right)

- Number of times button (left or right) changed (this is a new variable not used before)

- Mean time between button presses

- Standard deviation of time between button presses Physical variables:

- Number of hits (arrow moved in the intended direction). For the middle intention runs this was the negative number of steps away from the middle.

- Cumulative number of steps away from target

- Auto-correlation

- Number of cheer sounds (this is a new variable not used before)

- Standard deviation of the 80 raw random generator numbers 
The 5 psychological and physical variables from the nine runs yielded 45 psychological and 45 physical variables that were correlated against each other to yield $45 \times 45=2,025$ correlation factors. Each correlation factor was calculated across all participants. As mentioned above, for the main result of this study, only those correlations were considered that were based on time-forward correlations, i.e., only correlating psychological variables with physical variables from a later run. This resulted in 900 correlation factors remaining. While von Lucadou prefers to use all correlation factors, this author prefers the set reduced to time-forward correlations (i.e., psychological variables obtained before physical ones), to principally exclude the possibility of feedback-induced causal correlations as discussed above.

After the matrix elements (the correlation factors) were obtained, a single test statistic was calculated for the matrix, combining all individual correlation factors. For the analysis described here, the absolute values of all correlation factors in the matrix were cubed and then the mean value of these cubed factors was computed. This way of analyzing the data is different from the method used by von Lucadou (1986, 1991, 2006) and Walach et al. (2020): They used the number of 'significant' correlations in the matrix as the test statistic. That method has the potential disadvantage, though, that not all matrix elements contribute to the result. Analysis of existing matrix experiments with both test statistics (performed by this author) show that the mean sum of absolute correlation factors cubed seems to be at least 'as good' as the alternative used by von Lucadou, i.e., yields results that are at least as significant. This was tested on the data of the experiment reported in Walach et al. (2020). A similar test statistic has been used by Jolij and Bierman (2019), using the mean of squared correlation factors.

After determining the test statistic, in a second step the statistical significance of the obtained value was evaluated. This was done by empirically estimating the statistical background distribution (i.e., the expected distribution of results from an ensemble of many random representations of the experiment) using permutations of the psychological variables of participants against the physical variables of other participants. The test statistic for the unpermuted data (the main experimental result) was then compared to the distribution of test statistics from the permuted data. A $p$-value was calculated by dividing 
the number of higher test statistics (higher correlations) by the total number of test statistics obtained for the background. A one-sided hypothesis was used that the experimental data without permutations would show more and/or stronger correlations than most test statistics of the background distribution. The background distribution can also be simulated using artificial data as an alternative method. If both methods agree in their result, this can be taken as a strong argument for the validity of the estimated background, as pointed out by Grote (2017).

\section{RESULTS}

\section{Experiment 1}

The above pre-registered analysis (Experiment 1 ) was unblinded live during a presentation at the PA convention in Paris on July 6, 2019.

Figure 3 shows the main result against the background distribution that has been obtained by multiple permutations of the experimental dataset. The figure of merit falls well within the background distribution with $p=0.76$. The three control datasets obtained shortly

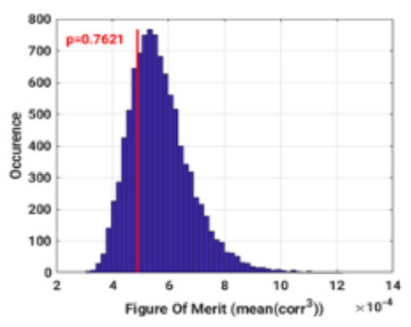

Figure 3. The main result obtained during the 'opening of the box event' at the 2019 PA convention. The graph shows the statistical background distribution obtained with 10,000 permutations of psychological vs. physical data (blue columns). The $x$-axis denotes the mean of all absolute values of cubed correlation factors in the matrix. The $y$-axis denotes the number of occurrences within the 10,000 permutations. The red vertical line shows the unpermuted experimental data. It falls well within the background distribution with a statistical value with a one-sided $p$-value of $p=.76$. 
before and after the experimental data (given in this order) also fall within the background distribution with $p$-values of $p=0.22, p=0.74$, and $p=0.08$, respectively (not shown in the graph).

Figure 4 shows the correlation matrices for one of the control datasets (top graph) and for the main experimental data (bottom graph). Notably, there seem to be more correlations close to the diagonal in
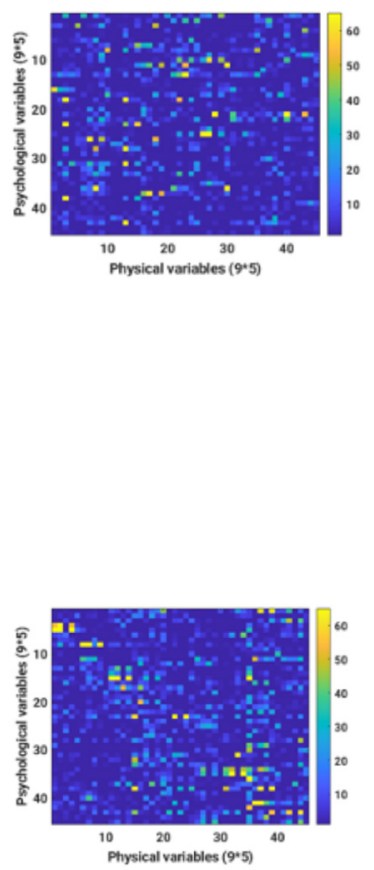

Figure 4. Correlation matrix for a control condition (top graph) and for the experimental results (bottom graph). The color code denotes the cubed absolute correlation factors (times 10,000 in units of the color code scales to the right of the graphs). Higher correlations can be observed close to the diagonal of the experimental matrix on the bottom graph. These are very likely causal correlations, which in any case had been excluded from the preplanned main statistical analysis. 
the experimental data matrix on the bottom graph. These are very likely due to causal correlations and are excluded from the main statistical analysis, which uses only the upper right part of the matrix. ${ }^{2}$

However, when including the full matrix in the analysis, including the diagonal elements, the figure of merit of the experimental data results in a probability of $p=0.17$ to have occurred by chance under a null hypothesis. So even when including the potential causal correlations, the result is not significant.

When splitting the main analysis (for the upper matrix) into the two types of random data used, the $p$-values are $p=0.5876$ for the real-time random data (RT-bits), and $p=0.4321$ for the XOR'd and prerecorded random data (XPR-bits). This result is commented on below.

\section{Experiment 2}

The analysis for Experiment 2 (the correlation between questionnaires and psi task) was unblinded in the sole presence of the author on September 15, 2019.

Figure 5 shows the result against the background distribution that has been obtained by multiple permutations of the experimental dataset.

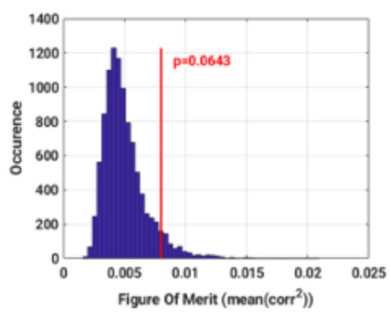

Figure 5. Result of Experiment 2 as obtained during the 'opening of the box'. The graph shows the statistical background distribution obtained with 10,000 permutations of psychological data of the questionnaires (50 variables) against physical data of the psi-task ( 1 variable). The $x$-axis denotes the mean of all (50) squared correlation factors. The $y$-axis denotes the number of occurrences within the 10,000 permutations. The red line shows the unpermuted experimental data. It results in a one-sided $p$-value of $p=.064$. 
The figure of merit falls slightly to the right side of the background distribution with a probability of $p=0.064$ to have occurred by chance under a null hypothesis. The three control datasets obtained shortly before and after the experimental data fall within the background distribution with $p$-values of $p=.61, p=.53$, and $p=.19$, respectively (not shown in the graph). When splitting this analysis into the two types of random data used, the $p$-values are $p=.1448$ for the real-time random data (RT-bits), and $p=.1678$ for the XOR'd and pre-recorded random data (XPR-bits). This difference apparently is insignificant.

\section{Post Hoc Analyses}

Since one emphasis of this study was to use a blind analysis protocol, no particular post hoc analysis was planned. However, blind analysis does not exclude post hoc analysis in principle, which can range from consistency checks to exploratory analysis in search for novel effects in the data. Blind analysis just helps to draw a very clear line of distinction between preplanned and post hoc analyses.

Triggered by the first of the additional observations as reported below, it was decided to calculate the simple psi-task related outcome of how successful the 200 participants were in 'shifting' the arrow in the display in the instructed left or right direction. It was found that the participants were 'successful' in the intended direction (hits) with a surplus of 138 bits out of $N=96,000$ trials $(200 \times 6 \times 80)$. This corresponds to a probability of $p=0.064$ (one-sided) to have occurred by chance under a null hypothesis. Note that the standard deviation $(S D)$ for the underlying Markov chain is $S D=\operatorname{sqrt}(N / 12)$ and not the usual $S D=\operatorname{sqrt}(N / 4)$ as for independent random bits (see von Lucadou, 1986). Also note that the numerical value for this probability (.064) is the same as the above reported for the correlation analysis. This is pure coincidence, since the underlying degrees of freedom are completely independent in both cases (!).

The split analysis for the two types of random data (for the above analysis) yielded $p=.111$ for the real-time data and $p=.107$ for the XOR'd and pre-recorded data. Apparently, both of these contributed about equally to the combined result.

Another post hoc analysis was to run the analysis for the second experiment, also using the absolute value of correlation factor cubed, 
instead of squared. The result was exactly the same, i.e., a $p$-value of $p$ $=.064$. This nicely demonstrates the robustness of this figure of merit (at least for these data).

Further, the 50 psychological variables of the questionnaire were also correlated with the $5^{\star} 9$ physical variables as used for the analysis of the main experiment. This more resembles the way the data was analyzed for the early CMM experiments, as by von Lucadou (1986, 1991). The result was not significant with a $p$-value of $p=.647$.

\section{SOME ADDITIONAL OBSERVATIONS: TRICKSTER AT WORK?}

I would like to report here three occurrences that happened around the execution of the experiment and the testing and execution of the analysis, that I found quite remarkable. I would not classify these as 'post hoc' analyses, but rather as observations that occurred, without deliberately looking for them.

The first occurrence was the experimental result of an 8-year-old boy, who performed the experiment as the only person other than the regular cohort of 200 participants. Since the experiment required the filling out of a questionnaire and the signing of a consent form, it was only suitable for adults. However, during my presence at the house of his parents, the boy insisted on performing the experiment. So I let him have his way, while deciding that his data would not enter the analysis. At the end of his session, the boy had reached the most extreme result of the main psi task (the number of hits), compared with all 200 other participants. His total score was -19 with a two-sided probability of this or a more extreme result to have occurred by chance (under a null hypothesis) of $p=.0038$. Obviously, if 200 participants do such an experiment, the occurrence of one such result is not surprising at all. The surprise lies in the fact that this result was obtained by the only non-regular performer of the experiment, who happened to be a child.

The second occurrence was related to the differential analysis, comparing the experimental results for the two cohorts of data using either the real-time RNG (RT-bits) data or the XOR'd and prerecorded RNG data (XPR-bits). This code for this analysis was tested using artificial pseudo-random data generated with Matlab. The first three tests of this analysis code yielded $p=.06, p=.95$, and $p=.0003$ for the 
results (or more extreme ones) to have occurred by chance. As a sort of memo, Figure 6 shows the last result as it was first obtained. The ' $p=0$ ' result of this test came from the limited number of permutations of $n=1,000$ used for estimating the statistical background of the test data. In a subsequent run using $n=10,000$ permutations for the same test data, the more accurate estimate of $p=.0003$ as reported above was obtained. The combined $p$-value for the three extreme results is of the order $p \sim .0001$. Further tests with new artificial data converged to a uniform distribution of $p$-values as expected. So while this was a test run for the analysis code, subsequently nothing was found wrong with the code. Given this expected performance of the analysis code, I found the initial results quite surprising. Similar surprises had been encountered in other work reported by Grote (2017).

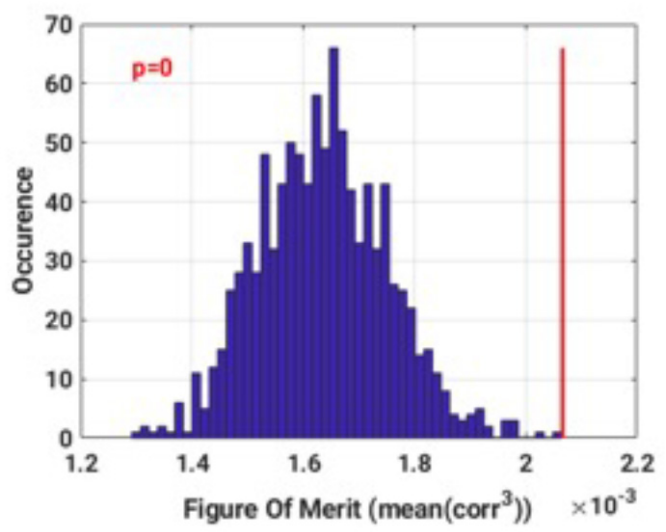

Figure 6. Result of third test run for the split RNG analysis. The author was struck by the extreme result, with subsequent results converging to the expected uniform distribution.

The third occurrence was related to finally performing the differential analysis on the unpermuted data, once the testing of the code for the split RNG analysis had been completed. Figure 7 shows the two histograms of background distribution and the results for the two random event groups. After I had first produced the graph on the top, I thought that the composition of digits of the obtained $p$-value of $p=.5876$ looked 'funny', like a sequence of numbers after the decimal 
point, though shifted by one digit. While briefly wondering about this, I produced the graph on the bottom next, which, to my surprise, resulted in a $p$-value of $p=.4321$. Note that these two $p$-values are completely independent of each other, since they represent the data of the main experiment, split into two independent groups, according to which type of random event was used.

\section{DISCUSSION}
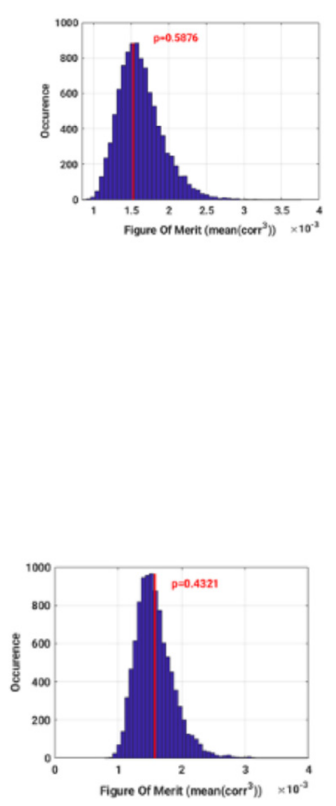

Figure 7. Result of the main experiment as split for the two types of RNG data used: Using the real-time data (top) and the XOR'd and prerecorded data (bottom). The author was struck by the 'funny' numbers of the estimated probabilities. 
Each reader may draw their own conclusions, but the experiments and their results reported here may merit discussion of several topics: 1) The conceptual replication of a CMM experiment using a blind analysis protocol; 2) The conceptual replication of a CMM-similar experiment correlating questionnaires with a single psi task, also using a blind analysis protocol; 3) A post hoc finding of marginal significance of the main psi task and its relation to the replication problem and the idea of the CMM experiment; 4) Trickster-like properties of the experimental outcomes and incidental observations; and 5) some thoughts about future research.

1) The conceptual replication of the CMM-type experiment of the type performed before (von Lucadou, 2006; Walach et al., 2020) (Experiment 1 as reported above) has yielded no evidence of anomalous correlations. While the methodology of using blind analysis was slightly stricter than the methodology in von Lucadou's and Walach et al.'s experiments, I do not believe that this was a decisive factor in this outcome. If we do not question the positive results reported by von Lucadou (1986, 1991, 2006) and Walach et al. (2020), I would interpret the null result of the experiment reported in this study as either the result of a decline of effects in repeated parapsychology experiments, or as a sign that I am not a very psi-conductive experimenter (at least for this experiment). The latter would pertain to the hypothesis that experimenters may have different levels of 'psi-conductivity', as has been observed by different researchers. Both of these interpretations touch upon profound problems in parapsychological research.

If experimenter-psi effects are taken into account (see Palmer \& Millar, 2015, for a more recent overview), then in addition one may also consider the audience that observed the unblinding of the main experiment during the presentation at the PA convention in Paris. With some humor I would say I am a bit disappointed by the psi performance of this particular, select audience in 'influencing' the outcome of the main experiment. In a snap poll, about half of the audience expressed the opinion that the result of the study was not yet determined at the moment before the unblinding of the analysis, leaving room for the audience to 'influence' the imminent outcome. In their defense one may argue that the total person-hours of the audience are significantly fewer $(\sim 100)$ than the person hours the author invested 
in this study ( 600). This could provide a simple numerical measure of a psi effort, while other concepts such as 'involvement' and 'motivation' are harder to quantify.

While the experimenter psi hypothesis is often neglected in parapsychological research, perhaps to no small degree because it renders research even more difficult in terms of reproducibility and other factors, a correlation experiment seems to be particularly prone to experimenter-psi, if it exists. This is because in a correlation experiment, no single individual participant can be 'successful' on his or her own. The result of the experiment is always one (or many) correlation factor(s) obtained across an ensemble of participants. For a single participant the result would simply not be defined. The group of participants can generate the effect under study only as an ensemble. In this context, it's completely unclear what the role of a 'gifted subject' may be. One can speculate that if experimenter-psi exists (and why would it not under a psi hypothesis?), then a correlation-type experiment is particularly prone to experimenter-psi.

2) The second experiment in this study (Experiment 2), a conceptual replication of the work reported by Jolij and Bierman (2019), yielded marginal evidence $(p=.064$ ) of anomalous connectivity-of the same order of magnitude as found in Jolij and Bierman. It is worth noting that this way of performing a CMM-like experiment is closer to the early experiments of von Lucadou $(1986,1991)$ where questionnaires were also used. I would not claim here that a new experimental paradigm has been found, but rather make note that this is only the first replication of the work by Jolij and Bierman, and effects may decay in the future. Taking the marginal evidence seriously, another interpretation would be that of a Trickster-like result, where the effect went back to where it was found in the past, i.e., in the correlation studies using questionnaires as reported by von Lucadou (1986, 1991). Note though that only one physical variable was used in the planned analysis presented here, and the correlation with all physical variables was found not to be significant (as reported above).

If one is inclined to interpret the result of $p=.064$ as an anomaly, it is interesting to note that no difference between the two types of random source has been found, which implies that the result was obtained including some level of 'retro-pk'-like phenomenon (see 
for example Schmidt, 1976) and was also independent of the type or complexity of random event generator (Schmidt, 1987).

3) The posthoc result of $p=.064$ (the same number as above, but a different result!) for the main psi task of 'influencing' the leftright direction of the observed arrow by the participants may be a chance result, of course. It is remarkable though that such an effect showed up in a place where it was not expected, since the whole CMM idea was developed around the notion that psi would not act in a 'signal-like' fashion such as to 'influence' the outcome of RNGs in a particular direction. If one interprets the observed $p=.064$ deviation as the hint of an anomaly, then the whole history has come full circle: An effect in a simpler RNG-PK 'influence' type of experiment was observed, but not in the more complex CMM-type analysis. Again, this has more of a Trickster quality than anything else. The CMM method was an ingenious idea to reliably observe anomalous connections in data by way of multiple correlations. However, we may be facing Trickster qualities that will always be a bit more ingenious. This is a conclusion that certainly would concur with J. P. Hansen's analysis of parapsychological research (Hansen, 2001), and that has also been exposed by Kennedy (Kennedy, 2003).

4) Further to the Trickster-like observation in 3), the three reported incidental observations can be seen as pure chance and would probably be dismissed and not be reported by most researchers, certainly in mainstream academia. In the context of a psi experiment, to me they had a very special quality, and I am more inclined to interpret them as another Trickster manifestation: While psi effects have been sought in a carefully planned and executed experiment, they have been found at the edges of this endeavor, in unexpected places. In addition to its Trickster quality, this sort of 'displacement' effect has also been described as the 'capricious' or 'unsustainable' nature of psi (see Kennedy, 2003, and references therein).

5) My main conclusion and suspicion is that the CMM idea will not escape the replication problem in parapsychology. Another possible interpretation could come from dominant psi-experimenter effects. Under a psi hypothesis, psi-experimenter effects need to be taken seriously. This has been pointed out by many (see again Palmer \& Millar, 2015, p. 293, for an overview), but it still is not a theme frequently 
adhered to across the board. In addition to psi-experimenter effects, the Trickster theme (which obviously would also play into psi-experimenter effects) may be more ubiquitous than existing scientific literature would make us believe.

The experiments reported here have spanned methodology from the extremely controlled (blind analysis) to the anecdotal (subjectively 'funny' or surprising incidences), and I believe the full breadth of these methodologies is needed to shed light on psi in the experimental domain. Obviously, it would make not much sense now to propose an experiment where we look for a Trickster effect, such as for odd statistical results when testing analysis code. This would lead to a regression ad infinitum. In a sense this is what the CMM idea was trying. We may as well continue to do so, but I believe it will be important to pay attention to the more subjective qualities of such experiments as well. I strongly think that experimental parapsychology research should be rigorous (to not fool ourselves) and descriptive (in a subjective experience sense) at the same time. A planned experiment then also resembles something of a single case study in the field. This avenue has been less explored, but the recent work by Herb Mertz is an excellent example in this category (2020).

\section{NOTES}

1 The experiments published by Walter von Lucadou (von Lucadou 1986, 1991, 2006) had not taken into account internal correlations between psychological variables and thus overestimated the statistical significance of the results. The method proposed and applied by this author, to use simulations or appropriate permutations to estimate the statistical significance, was deemed 'conservative' by other researchers (Walach et al., 2020), citing work by Calude and Longo (2017). This author thinks citing Calude and Longo in this context misses the point. The fact that (nearly) any correlations can be found in a (nearly) infinite amount of data is unrelated to the question of statistical significance.

2 When observing the correlations of psychological and physical variables from the same run (the 9 times $5 \times 5$ pixels on the diagonal of the matrix), the physical variables tending to showing higher correlations are 1, 2, and 4, predominantly with psychological vari- 
ables 4 and 5. Physical variables 1, 2, and 4 are related to the 'success' of the participant, and psychological variables 4 and 5 are the mean and variance of the times to press buttons. A causal explanation of increased correlations may well be the longer time and variance participants may have used for pressing buttons when being 'successful' and hearing more cheer sounds. (The cheering sounds last longer than just the 'success in intended direction' sound.)

\section{ACKNOWLEDGMENTS}

I thank the BIAL foundation for support of this work under grant number 161/19. I also thank Eberhard Bauer, Bettina Grote, Karolina Kirmse, and Nikolaus von Stillfried for valuable comments on the manuscript and Walter von Lucadou for his enthusiasm and tireless discussions on the topic. I thank the Max Planck Society for endorsing this work via their ethics committee. No other resources of the Max Planck Society have been used for this study.

\section{REFERENCES}

Bierman, D. J., Spottiswoode, J. P., \& Bijl, A. (2016). Testing for questionable research practices in a meta-analysis: An example from experimental parapsychology. PLOS One, 11(5), e0153049. https://doi.org/10.1371/journal. pone.0153049

Calude, C. S., \& Longo, G. (2017). The deluge of spurious correlations in big data. Foundations of Science, 22(3), 595-612.

Filk, T., \& Römer, H. (2011). Generalized quantum theory: Overview and latest developments. Axiomathes, 21, 211-230. https://arxiv.org/abs/1202.1659 https://doi.org/10.1007/s10516-010-9136-6

Grote, H. (2015). A correlation study between human intention and the output of a binary random event generator. Journal of Scientific Exploration, 29(2), 265-290.

Grote, H. (2017). Multiple-analysis correlation study between human psychological variables and binary random events. Journal of Scientific Exploration, 31(2), 231-254.

Hansen, G. P. (2001). The trickster and the paranormal. Xlibris. ISBN 1-4010-0082-7.

Jolij, J., \& Bierman, D. (2019). Two attempted retro-priming replications show theory-relevant anomalous connectivity. Journal of Scientific Exploration, $33(1), 43-60$.

Kennedy, J. E. (2003). The capricious, actively evasive, unsustainable nature of psi: A summary and hypotheses. Journal of Parapsychology, 67(1), 53-74.

Klein, J. R., \& Roodman, A. (2005). Blind analysis in nuclear and particle physics. 
Annual Review of Nuclear and Particle Science, 55, 141-163. https://doi. org/10.1146/annurev.nucl.55.090704.151521

MacCoun, R., \& Perlmutter, S. (2015). Blind analysis: Hide results to seek the truth. Nature, 526(7572), 187-189.

Marsaglia, G. (1995). The Marsaglia random number CDROM including the Diehard battery of tests of randomness. https://web.archive.org/ web/20160125103112/http://stat.fsu.edu/pub/diehard/

Matsumoto, M., \& Nishimura, T. (1998). Mersenne twister: A 623-dimensionally equidistributed uniform pseudo-random number generator. ACM Transactions on Modeling and Computer Simulation, 8(1), 3-30. https://doi. org/10.1145/272991.272995

Mertz, H. (2020). The selection effect: How consciousness shapes reality. Penn Wolcott Press. ISBN 1733508007.

Palmer, J., \& Millar, B. (2015). Experimenter effects in parapsychology research. In E. Cardeña, J. Palmer, \& D. Marcusson-Clavertz (Eds.), Parapsychology: A handbook for the $21^{\text {st }}$ century. Mc Farland.

Schmidt, H. (1976). PK effect on pre-recorded targets. Journal for the American Society for Psychical Research, 70, 267-291.

Schmidt, H. (1987). The strange properties of psychokinesis. Journal of Scientific Exploration, 1(2), 108-118.

von Lucadou, W. (1986). Experimentelle Untersuchungen zur Beeinflussbarkeit von stochastischen quantenphysikalischen Systemen durch den Beobachter. H.-A. Herchen Verlag.

von Lucadou, W. (1991). Locating Psi-bursts: Correlations between psychological characteristics of observers and observed quantum physical fluctuations. In D. L. Delanoy (Ed.), The Parapsychological Association $34^{\text {th }}$ Annual Convention Proceedings of Presented Papers (pp. 265-281).

von Lucadou, W. (1995). The model of pragmatic information (MPI). European Journal of Parapsychology, 11, 58-75.

von Lucadou, W. (2006). Self-organization of temporal structures-A possible solution for the intervention problem. In D. P. Sheehan (Ed.), Frontiers of time. Retrocausation-Experiment and theory. AIP Conference Proceedings, 863, 293-315 (San Diego, CA, June 20-22). https://doi.org/10.1063/1.2388760 Wagenmakers, E.-J., Wetzels, R., Borsboom, D., Kievit, R., \& van der Maas, H. L. J. (2015). A skeptical eye on psi. In E. May \& S. Marwaha (Eds.). Extrasensory perception: Support, skepticism, and science (pp. 153-176). Praeger.

Walach, H., Horan, M., Hinterberger, T., \& von Lucadou, W. (2020). Evidence for anomalistic correlations between human behavior and a random event generator: Result of an independent replication of a micro-PK experiment. Psychology of Consciousness: Theory, Research, and Practice, 7(2), 173-188. https://doi.org/10.1037/cnsoooo199 